\title{
Multiuser Diversity - Multiplexing Tradeoff in MIMO Broadcast Channels with Limited Feedback
}

\author{
Marios Kountouris ${ }^{\dagger}$, Ruben de Francisco ${ }^{\ddagger}$, David Gesbert ${ }^{\ddagger}$, \\ Dirk T. M. Slock ${ }^{\ddagger}$ and Thomas Sälzer ${ }^{\dagger}$ \\ ${ }^{\dagger}$ France Telecom Research and Development, Issy-les-Moulineaux, France \\ \{marios.kountouris, thomas.salzer\}@ orange-ftgroup.com \\ ${ }^{\ddagger}$ Eurecom Institute, Sophia-Antipolis, France \\ \{defranci, gesbert, slock\}@eurecom.fr
}

\begin{abstract}
We consider joint scheduling and beamforming in a broadcast channel with multiple antennas at the transmitter and a single antenna at the mobile receiver. Perfect channel knowledge is assumed to be available at the receiver while the transmitter is provided with partial channel state information (CSIT) through a limited rate feedback channel. Each user feeds back quantized information regarding the channel vector direction (from a codebook) and a quantized (scalar) channel quality indicator. We identify the tradeoff between multiuser diversity and spatial multiplexing gain given a limited amount of feedback bits. Scaling laws of the above parameters are derived in order to achieve a target rate performance. Our results reveal useful design guidelines for the split of feedback bits for channel quantization and channel quality.
\end{abstract}

\section{INTRODUCTION}

Multiple-input multiple-output (MIMO) communication systems have the potential to offer high spectral efficiency as well as link reliability. In MIMO broadcast channels, it was shown [1], [2] that the capacity can be boosted by exploiting the spatial multiplexing capability of transmit antennas and transmit to multiple users simultaneously, by means of Space Division Multiple Access (SDMA), rather than trying to maximize the capacity of a single-user link.

As the capacity-achieving dirty paper coding (DPC) approach [3] is difficult to implement, many more practical downlink transmission techniques have been proposed. Downlink linear beamforming, although suboptimal, has been shown to achieve a large portion of DPC capacity, exhibiting reduced complexity [4], [5], [6]. Nevertheless, the capacity gain of multiuser MIMO systems seems to remain highly sensitive and dependent on the channel state information available at the transmitter (CSIT). If a base station with $M$ transmit antennas communicating with $K$ single-antenna receivers has perfect CSI, a multiplexing gain of $\min (M, K)$ can be achieved. The approximation of close to perfect CSI at the receiver (CSIR) is often reasonable, especially for downlink channels, where a common pilot can be employed for channel estimation by a large number of users. However, this assumption is unrealistic at the transmitter. Recently, it was shown that if the transmitter has imperfect channel knowledge, the full

The research of the authors at Eurecom was supported in part by the Eurecom Institute, and by the national RNRT project Lao Tseu. multiplexing gain is reduced at high SNR [7], whereas if there is complete lack of CSI knowledge, the multiplexing gain collapses to one [8]. Hence, as the broadcast channel's capacity is sensitive to the accuracy of CSIT, it is of particular interest to identify what kind of partial CSIT can be conveyed to the base station in order to achieve rates reasonably close to the optimum. The challenge consists in designing limited feedback strategies able to achieve spatial multiplexing gain and exploit multiuser diversity. These strategies have to be able to identify the appropriate users to be scheduled and enable the design of efficient beamforming techniques in order to provide near optimal capacity growth, while keeping the amount of feedback minimal.

It has been shown in MIMO point-to-point systems [9] that there exists a tradeoff between spatial multiplexing gain

$$
r=\lim _{P \rightarrow \infty} \frac{\mathcal{R}(P)}{\log P}
$$

and diversity gain

$$
-d=\lim _{P \rightarrow \infty} \frac{\mathcal{P}_{e}(P)}{\log P}
$$

which is defined in the high SNR regime, given the system rate $\mathcal{R}$ and average error probability $\mathcal{P}_{e}$. This tradeoff is due to the fact that there is a certain number of degrees of freedom in the MIMO channel that need to be shared in order to achieve diversity or increase the transmission rate. In MIMO broadcast channels with user scheduling, there is in addition multiuser diversity gain

$$
m=\lim _{K \rightarrow \infty} \frac{\mathcal{R}(P, K)}{r \log \log K}
$$

The multiuser diversity differs from single-user diversity in the sense that the latter refers to the ability for the multiple antennas to receive the same information across different paths, while in multiuser systems, different information is transmitted and received by different users. The multiuser diversity gain increases with the number of active users in the cell, while the available multiplexing gain remains $\min (M, K)$, regardless of the value of $K$. Hence, with full CSIT both multiuser diversity and multiplexing gain can be attained since they scale with 
different magnitudes, $K$ and SNR respectively. The tradeoff appears when we consider a system with limited feedback rate, in which each mobile is allowed to feed back a finite number of bits.

In point-to-point MIMO systems, the effect of limited feedback on the system rate is less severe than in MIMO broadcast channels. It has been shown in [10] that even a few feedback bits can provide performance close to that with full CSIT, achieving full multiplexing gain. However, as it has been recently shown in [11], the level of CSIT critically affects the multiplexing gain of the MIMO broadcast channel. In order to achieve full multiplexing gain, the feedback load per user must increase approximately linearly with the number of transmit antennas and the number of feedback bits per mobile must increase linearly with the SNR (in $\mathrm{dB}$ ). Hence, appropriate feedback load scaling in terms of channel directional information (CDI) is needed to achieve full multiplexing gain. On the other hand, CDI can not exploit multiuser diversity gain in a multiuser context with $K \geq M$. In this situation, channel gain information (CGI) at the transmitter becomes necessary in order to perform efficient user selection. However, since the available feedback rate is finite, the amount of bits used for CSIT quantization has to be shared for both CDI and CGI quantization. While CDI quantization incurs in loss of multiplexing gain, CGI quantization leads to a degradation of the multiuser diversity benefit.

In this paper, a tradeoff between multiuser diversity and multiplexing gain in MIMO broadcast channels with limited feedback is presented. The problem of feedback splitting for channel directional information (CDI) and channel gain information (CGI) is introduced and useful feedback design guidelines are provided. A particular case is studied, in order to illustrate the importance of this tradeoff, providing extensive and intuitive simulation results.

\section{System Model}

We consider a multiple antenna broadcast channel consisting of $M$ antennas at the transmitter and $K \geq M$ singleantenna receivers. The received signal $y_{k}$ of the $k$-th user is mathematically described as

$$
y_{k}=\mathbf{h}_{k}^{H} \mathbf{x}+n_{k}, \quad k=1, \ldots, K
$$

where $\mathbf{x} \in \mathbb{C}^{M \times 1}$ is the transmitted signal, $\mathbf{h}_{k} \in \mathbb{C}^{M \times 1}$ is the channel vector, and $n_{k}$ is additive white Gaussian noise at receiver $k$. We assume that each of the receivers has perfect and instantaneous knowledge of its own channel $\mathbf{h}_{k}$, and that $n_{k}$ is independent and identically distributed (i.i.d.) circularly symmetric complex Gaussian with zero mean and unit variance. The transmitted signal is subject to an average transmit power constraint, i.e. $\mathbb{E}\left\{\|\mathbf{x}\|^{2}\right\}=P$, and all users have the same average signal-to-noise ratio (SNR). We consider an i.i.d. block Rayleigh flat fading channel, whose parameters are considered invariant during each coded block, but are allowed to vary independently from block to block.

\section{Finite Sum Rate Feedback Model}

In this section, we present a general framework which is referred to as finite sum rate feedback model. In this context, each receiver $k$ is constrained to have a limited total number of feedback bits $B_{t o t}$. From this total amount of bits, $B_{1}$ bits are used to quantize the channel direction information (CDI) $\overline{\mathbf{h}}=\mathbf{h} /\|\mathbf{h}\|$ based on a predetermined codebook, and $B_{2}$ bits are used for scalar quantization of real-valued channel gain information (CGI). This model is depicted in Fig. 1.

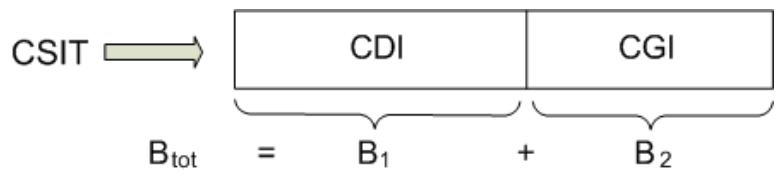

Fig. 1. Finite Sum Rate Feedback Model.

Channel directional information can be used to achieve full multiplexing gain when the feedback load $B_{1}$ scales appropriately [11]. In a multiuser context with $K>M$, CDI does not provide any information on users' channel gains, thus not being sufficient for efficient user selection failing to exploit multiuser diversity gain. Hence, additional instantaneous lowrate channel gain information is required as an indicator of the channel quality. The goal of this paper is to reveal the interplay between $K$, average SNR and feedback load $B_{1}$ and $B_{2}$, in order to exploit in the best possible way the degrees of freedom available in a multiuser MIMO downlink, i.e., the spatial multiplexing and multiuser diversity gain. The sum feedback rate constraint per user $\left(B_{t o t}\right)$ results to a tradeoff between multiuser diversity and multiplexing gain, and we focus on characterizing it by identifying the optimal feedback rate allocation (split) in order to exploit both gains. Plainly speaking, we intend to determine how many feedback bits are CDI and CGI worth.

\section{A. Channel Direction Quantization}

We consider a quantization codebook $\mathcal{V}_{k}=$ $\left\{\mathbf{v}_{k 1}, \mathbf{v}_{k 2}, \ldots, \mathbf{v}_{k N_{1}}\right\}$ containing $N_{1}=2^{B_{1}}$ unit norm vectors $\mathbf{v}_{k i} \in \mathbb{C}^{M}$, for $i=1, \ldots, N_{1}$, which is assumed to be known to both the $k$-th receiver and the transmitter. In the most general case, the mobile terminals can have different codebooks, generated through random unitary rotation of a common, general codebook $\mathcal{V}_{g}$ known at both ends of the link. Based on the channel realization, the receiver selects its 'best' vector from the codebook, i.e. the codeword that optimizes a certain cost function. Here, we assume that each receiver quantizes its channel to the vector that maximizes the following inner product [11], [12], [13], [14], i.e.,

$$
\hat{\mathbf{h}}_{k}=\mathbf{v}_{k n}=\arg \max _{\mathbf{v}_{k i} \in \mathcal{V}_{k}}\left|\overline{\mathbf{h}}_{k}^{H} \mathbf{v}_{k i}\right|^{2}
$$

where $\hat{\mathbf{h}}_{k}$ is the $k$-th user channel quantization, constrained to be unit-norm and invariant to arbitrary phase rotation. Each user sends the corresponding quantization index $n$ back to the transmitter through an error-free, and zero-delay feedback channel using $B_{1}=\left\lceil\log _{2} N_{1}\right\rceil$ bits. 


\section{B. Channel Gain Quantization}

As channel quality indicator, we consider instantaneous scalar feedback, denoted as $\xi_{k}$, which can take on various forms and is evidently a certain function of the current channel realization $\mathbf{h}_{k}$ (i.e., $\xi_{k}=f\left(\mathbf{h}_{k}\right)$ ). We assume that $\xi_{k}$ are i.i.d. random variables with probability density function (pdf) $f_{\xi}(\xi)$.

Let $\xi$ and $\mathcal{Q}(\xi)$ denote the input and the output values of quantizer $\mathcal{Q}(\cdot)$. Let $\mathcal{X}=\left\{q_{0}<q_{1}<\ldots<q_{N_{2}}\right\}$ be the input decision levels and let $\mathcal{Y}=\left\{\xi_{q_{0}}<\xi_{q_{1}}<\ldots<\xi_{q_{N_{2}-1}}\right\}$ be the output representative levels (reconstruction values) of an $N_{2}$-level quantizer $\mathcal{Q}(\cdot)$ defined as:

$$
\mathcal{Q}(\xi)=\xi_{q_{i}} \text { if } q_{i} \leq \xi<q_{i+1} \quad 0 \leq i \leq N_{2}-1
$$

with $q_{0}=0$ and $q_{N_{2}}=\infty$. A partition region (quantization level) is defined as $Q_{i}=\left[q_{i}, q_{i+1}\right), 0 \leq i \leq N_{2}-1$. Each user sends the corresponding quantization level index $i$ back to the transmitter using $B_{2}=\left\lceil\log _{2} N_{2}\right\rceil$ bits. In order to minimize the outage probability, we assume the following conservative but reliable quantization rule $\xi_{q_{i}}=q_{i}$.

\section{Problem Formulation}

Our objective is to dynamically allocate bits to CDI and CGI feedback (as shown in Fig. 1) given a total amount of feedback bits $B_{t o t}$, so that the capacity of the multiuser MIMO downlink $\mathcal{C}\left(B_{1}, B_{2}\right)$ is maximized. In the described finite sum rate feedback model, the optimal feedback rate allocation that maximizes the capacity can be formulated in the following constrained optimization problem:

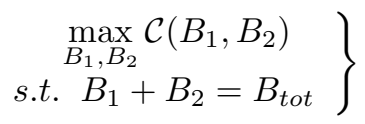

Let $\mathcal{W}$ be the event that a user $k$ is selected for transmission among $K$ users. Using the analysis of [15], we calculate the probability of the above event conditioned on the fact that $\xi$ falls into the quantization level $Q_{j}$

$$
\operatorname{Pr}\left(\mathcal{W} \mid \xi \in Q_{j}\right)=\sum_{m=0}^{K} \frac{1}{m+1} \cdot\left(\begin{array}{c}
K-1 \\
m
\end{array}\right) \cdot \mathcal{P}_{1} \cdot \mathcal{P}_{2}
$$

where

$\mathcal{P}_{1}=\operatorname{Pr}\left\{m\right.$ users other than user $\left.k \in Q_{j}\right\}=\left(\operatorname{Pr}\left(\xi \in Q_{j}\right)\right)^{m}$ and

$\mathcal{P}_{2}=\operatorname{Pr}\left\{(K-m-1)\right.$ users other than user $\left.k \in Q_{n}, n<j\right\}$

$$
=\left(\operatorname{Pr}\left(\xi \in \bigcup_{n<j} Q_{n}\right)\right)^{K-m-1}
$$

We assume here that if more than one user lie in $Q_{j}$, a random user is scheduled for transmission. Using that $\left(\operatorname{Pr}\left(\xi \in Q_{j}\right)\right)=$ $F_{\xi}\left(q_{j+1}\right)-F_{\xi}\left(q_{j}\right)$, and after some manipulations, one can show that

$$
\operatorname{Pr}\left(\mathcal{W} \mid \xi \in Q_{j}\right)=\frac{\left[F_{\xi}\left(q_{j+1}\right)\right]^{K}-\left[F_{\xi}\left(q_{j}\right)\right]^{K}}{K\left(F_{\xi}\left(q_{j+1}\right)-F_{\xi}\left(q_{j}\right)\right)}
$$

where $F_{\xi}(\cdot)$ is the cumulative distribution function (cdf) of the CGI.
In a system with joint downlink scheduling and beamforming with limited feedback, beamforming is performed based on quantized channel directions. Let's now assume that the quality indicator $\xi$ is a function of each user's SINR. In that case, the effect of CDI quantization will be reflected on the distribution of $\xi$. Hence, the CGI contains information both on channel gain and CDI quantization error. For instance, the value $\xi$ can be a lower/upper bound on the achievable SINR or even the achievable SINR value itself. Suppose now that the metric $\xi$ is a lower bound on the SINR. Then, the rate of the selected user $k, \mathcal{R}_{k}$ is given by

$$
\begin{gathered}
\mathcal{R}_{k} \geq \sum_{j=0}^{N_{2}-1} \int_{\xi \in Q_{j}} \operatorname{Pr}\left(\mathcal{W} \mid \xi \in Q_{j}\right) \log _{2}(1+\xi) f_{\xi}(\xi) d \xi \\
=\sum_{j=0}^{N_{2}-1} \int_{Q_{j}} \log _{2}(1+\xi) \cdot \frac{\left[F_{\xi}\left(q_{j+1}\right)\right]^{K}-\left[F_{\xi}\left(q_{j}\right)\right]^{K}}{K\left(F_{\xi}\left(q_{j+1}\right)-F_{\xi}\left(q_{j}\right)\right)} \cdot f_{\xi}(\xi) d \xi
\end{gathered}
$$

Let $\mathcal{S}$ be a set of scheduled users with cardinality $|\mathcal{S}|=M$. The system capacity $\mathcal{C}\left(B_{1}, B_{2}\right)$ can be lower bounded by

$$
\begin{gathered}
\mathcal{C}\left(B_{1}, B_{2}\right)=\sum_{k \in \mathcal{S}} \mathcal{R}_{k} \geq \\
\sum_{k \in \mathcal{S}} \sum_{j=0}^{2^{B_{2}}-1} \int_{Q_{j}} \log _{2}(1+\xi) \frac{\left[F_{\xi}\left(q_{j+1}\right)\right]^{K}-\left[F_{\xi}\left(q_{j}\right)\right]^{K}}{K\left(F_{\xi}\left(q_{j+1}\right)-F_{\xi}\left(q_{j}\right)\right)} f_{\xi}(\xi) d \xi
\end{gathered}
$$

where $B_{1}$ is contained both in $F_{\xi}(\xi)$ and $f_{\xi}(\xi)$.

From the above analysis, it can be seen that the optimization problem in (6) has no closed-form solution. Additionally, the solution depends on the quantization levels $q_{i}, 0 \leq i \leq N_{2}-1$ to be considered, thus different CGI quantization strategies will yield different solutions. To circumvent the complexity of numerical brute force optimization and the non-linearity of this optimization problem, numerical algorithms relying on dynamic programming and providing a global optimum can be used [16].

\section{Decoupled Feedback Optimization}

Instead of determining jointly the optimal feedback bit split, an approach of reduced complexity consists of decomposing the problem in a two-step procedure: we first find the optimal number of CDI bits required to guarantee full multiplexing gain, implying that the feedback load that is allocated to CGI is $B_{2}=\left(B_{t o t}-B_{1}\right)$, and optimizing the $2^{B_{2}}$ quantization levels by using (8).

We apply the finite sum rate feedback model in a scheme that performs zero-forcing beamforming on the channel quantizations available at the transmitter as a multiuser transmission strategy. Each user $k$ feeds back the index of its quantized channel direction (based on codebook $\mathcal{V}_{k}$ ), and the following quantized scalar feedback [17].

$$
\xi_{k}=\frac{P\left\|\mathbf{h}_{k}\right\|^{2} \cos ^{2} \phi_{k}}{P\left\|\mathbf{h}_{k}\right\|^{2} \sin ^{2} \phi_{k}+M}
$$


where $\phi_{k}=\angle\left(\hat{\mathbf{h}}_{k}, \overline{\mathbf{h}}_{k}\right)$. Note that a similar metric is also reported in [18], [19]. This type of CGI encapsulates information on the channel gain as well as the CDI quantization error, defined as $\sin ^{2} \phi_{k}=1-\left|\hat{\mathbf{h}}_{k}^{H} \overline{\mathbf{h}}_{k}\right|^{2}$, and it also provides an upper bound on each user's SINR. A system parameter $\epsilon$ that corresponds to the minimum required orthogonality factor between any pair of codebook vectors scheduled for transmission is defined, i.e. $\left|\hat{\mathbf{h}}_{i}^{H} \hat{\mathbf{h}}_{j}\right| \leq \epsilon, \forall i, j \in \mathcal{S}$. The users are selected based on the following scheduling algorithm [5], [6], [20]: the user with the highest $\xi_{k}$ value is first selected, and then the remaining $(M-1)$ users are chosen at each step as those having the highest $\xi_{k}$ values among the set of users whose channel quantizations are $\epsilon$-orthogonal to the quantized channels of previously selected users. Once $M \epsilon$-orthogonal users have been selected, the zero-forcing beamforming is applied based on the channel quantizations of the users selected for transmission.

Based on the asymptotic growth of (9) for large $K$ given in [21], we derive the scaling of CDI feedback load, which in turn determines the remaining CGI feedback bits. We define the power gap (per user) between the SINR of the above scheme, $S I N R_{I}$, and that of zero-forcing with perfect CSI, $S I N R_{Z F}$ as the ratio $\frac{S I N R_{I}}{S I N R_{Z F}}=\alpha$. Note that this power gap is translated to a rate gap. In order to achieve full multiplexing gain for finite $K$, the number of CDI bits $B_{1}$ per receiver $k$ should scale according to:

$$
B_{1}=(M-1) \log _{2}(P / M)-(1-\alpha) \log _{2} K-\log _{2} \kappa_{i-1}
$$

where $\kappa_{i-1}=\mathcal{K}_{i} / K$ is a constant capturing the multiuser diversity reduction at each step $i$ of the user selection algorithm (the number of users $\mathcal{K}_{i}$ from which $\xi_{k}$ is chosen is reduced at each step), due to the $\epsilon$-orthogonality constraint between scheduled users. As $\alpha<1$, having more users in the cell, a smaller number of feedback bits $B_{1}$ per user is required in order to achieve full multiplexing gain. For a system with $M=4$ antennas, $K=30$ users and SNR $=10 \mathrm{~dB}$, when a 3-dB SINR gap is considered, each user needs to feed back at least $B_{1}=9$ bits.

\section{A. Scaling of CDI feedback bits at high SNR regime}

The high SNR regime corresponds to the interferencelimited region, where the role of $\mathrm{CDI}$ is more critical due to the effect of quantization error [11]. As $P \rightarrow \infty$, the CGI becomes $\xi_{k}=\cot ^{2} \phi_{k}$, and based on asymptotic results of [21], we can show that for fixed $K$, the feedback load should scale as

$$
B_{1}=(M-1) \log _{2} P-\log _{2} K
$$

For instance, for a system with $M=4$ antennas, $\mathrm{SNR}=20 \mathrm{~dB}$ and $K=60$ users, $B_{1}=14$ bits are required to guarantee full multiplexing gain. As it was intuitively expected, the feedback load $B_{1}$ at high SNR is larger than that of (10). Thus, it is more beneficial to use more feedback bits on the quantization of channel direction $\left(B_{1}\right)$ at high SNR, and assign less bits for CGI $\left(B_{2}\right)$.

\section{Numerical Results}

In this section, we evaluate through simulations the sum rate performance of the scheme presented in Section $\mathrm{V}$, for $M=2$ transmit antennas and $\epsilon=0.4$. The total number of available feedback bits is $B_{t o t}=7$ bits. CGI quantization is performed through Lloyd's algorithm. Once both the input quantization levels $q_{i}$ and output representative levels $\xi_{q_{i}}$ are found, the quantizer sets $\xi_{q_{i}}=q_{i}, 0 \leq i \leq N_{2}-1$ in order to avoid outage events as discussed in Section III.

Fig. 2 and 3 show the sum rate as a function of the number of users for $\mathrm{SNR}=10 \mathrm{~dB}$ and $\mathrm{SNR}=20 \mathrm{~dB}$ respectively for different CDI and CGI feedback bit split. As expected, it is more beneficial to allocate more bits on channel direction quantization in a system with low number of active users. On the other hand, as the number of users increases, it becomes more beneficial to allocate bits on CGI quantization instead. The black curve $B_{1}=1$ bit corresponds to the random unitary beamforming for $M=2$ transmit antennas proposed in [22]. In a system with optimal quantization, i.e. matched to the pdf of the maximum CGI value among $K$ users, the amount of necessary quantization levels is reduced as the number of users in the cell increases. Thus, less amount of feedback bits is needed for CGI quantization in order to capture the multiuser diversity.

In Fig.4, the envelope of the curves in the two previous figures is shown, which corresponds to a system that chooses the best $B_{1} / B_{2}$ balance for each average SNR and $K$ pair. In this figure, we compare how this best pair of $\left(B_{1}, B_{2}\right)$ changes as the system average SNR increases. Both curves are divided in different regions, according to the optimal $\left(B_{1}, B_{2}\right)$ pair in each region. It can be seen that the optimal threshold for switching from $B_{1} \rightarrow B_{1}-1$ bits (and thus $B_{2} \rightarrow B_{2}+1$ ) is shifted to the right for higher average SNR values (upper curve). This means that as the average SNR increases, more bits should be allocated on channel direction information. Summarizing, given a pair of average SNR and $K$ values, there exists an optimal compromise of $B_{1}$ and $B_{2}$, given that $B_{t o t}=B_{1}+B_{2}$.

\section{CONCLUSION}

A MIMO broadcast channel with limited feedback has been considered, in which each user feeds back quantized information regarding its channel direction and a quantized real-valued channel quality indicator. We have formulated the problem of optimal feedback balancing in order to exploit spatial multiplexing gain and multiuser diversity gain under a sum feedback rate constraint. A low complexity approach has been discussed to illustrate the performance improvement of systems with optimally balanced feedback. The scaling of CDI feedback load in order to achieve full multiplexing gain is also provided, revealing an interesting interplay between the number of users, the average SNR and the number of feedback bits. 


\section{REFERENCES}

[1] G. Caire and S. Shamai, "On the achievable throughput of a multiantenna Gaussian broadcast channel," IEEE Trans. Inform. Theory, vol. 49, no. 7, pp. 1691-1706, July 2003.

[2] N. Jindal and A. Goldsmith, "Dirty paper coding vs. TDMA for MIMO broadcast channels," IEEE Trans. Inform. Theory, vol. 51, no. 5, pp. 1783-1794, May 2005.

[3] H. Weingarten, Y. Steinberg, and S. Shamai, "The capacity region of the Gaussian MIMO broadcast channel," in Proc. of 38th Conf. Inform. Sciences and Systems (CISS'04), Princeton, NJ, Mar. 2004.

[4] M. Sharif and B. Hassibi, "A comparison of time-sharing, DPC, and beamforming for MIMO broadcast channels with many users," submitted to IEEE Trans. on Commun., 2005.

[5] T. Yoo and A. Goldsmith, "On the optimality of multiantenna broadcast scheduling using zero-forcing beamforming," IEEE Journal on Sel. Areas in Commun. (JSAC), vol. 24, no. 3, pp. 528-541, Mar. 2006.

[6] G. Dimic and N. Sidiropoulos, "On downlink beamforming with greedy user selection: performance analysis and a simple new algorithm," IEEE Trans. Signal Processing, vol. 53, no. 10, pp. 3857-3868, Oct. 2005.

[7] A. Lapidoth and S. Shamai, "Collapse of degrees of freedom in MIMO Broadcast with finite precision CSI," in Proc. of 43rd Allerton Conf. on Commun., Control and Comput., Monticello, Illinois, USA, Sept. 2005.

[8] S. A. Jafar and A. Goldsmith, "Isotropic fading vector broadcast channels: The scalar upper bound and loss in degrees of freedom," IEEE Trans. Inform. Theory, vol. 51, no. 3, pp. 848-857, Mar. 2005.

[9] L. Zheng and D. N. Tse, "Diversity and multiplexing: A fundamental tradeoff in multiple antenna channels," IEEE Trans. Inform. Theory, vol. 49, no. 5, pp. 1073-1096, May 2003.

[10] D. J. Love, R. W. Heath, W. Santipach, and M. L. Honig, "What is the value of limited feedback for MIMO channels?" IEEE Comm. Magazine, vol. 42, no. 10, pp. 54-59, Oct. 2004.

[11] N. Jindal, "MIMO broadcast channels with finite rate feedback," in Proc. IEEE GLOBECOM'05, St. Louis, MO, Dec. 2005, pp. 1520-1524.

[12] D. J. Love, R. W. Heath, and T. Strohmer, "Grassmannian beamforming for multiple-input multiple-output wireless systems," IEEE Trans. Inform. Theory, vol. 49, no. 10, pp. 2735-2747, Oct. 2003.

[13] K. Mukkavilli, A. Sabharwal, E. Erkip, and B. Aazhang, "On beamforming with finite rate feedback in multiple-antenna systems," IEEE Trans. Inform. Theory, vol. 49, no. 10, pp. 2562-2579, Oct. 2003.

[14] P. Ding, D. Love, and M. Zoltowski, "Multiple antenna broadcast channels with shape feedback and limited feedback," submitted to IEEE Trans. Sig. Processing, 2005.

[15] F. Floren, O. Edfors, and B.-A. Molin, "The effect of feedback quantization on the throughput of a multiuser diversity scheme," in Proc. IEEE Global Telec. Conf. (GLOBECOM'03), San Francisco, U.S.A., Dec. 2003, pp. 497-501.

[16] M. Kountouris, R. de Francisco, D. Gesbert, D. T. M. Slock, and T. Salzer, "Multiuser Diversity - Multiplexing Tradeoff in MIMO Broadcast Channels with Finite Sum Rate Feedback," in preparation.

[17] — , "Efficient metric for Scheduling in MIMO Broadcast Channels with Limited Feedback," FT060303 - France Telecom R\&D internal report, Mar. 2006.

[18] N. Jindal, "Finite Rate Feedback MIMO Broadcast Channels," in Workshop on Inform. Theory and its Applications (ITA), UC San Diego, U.S.A. (invited paper), Feb. 2006.

[19] T.Yoo, N. Jindal, and A. Goldsmith, "Finite-rate feedback MIMO broadcast channels with a large number of users," in Proc. of IEEE Int. Symp. Inform. Theory (ISIT'06), Seattle, Washington, U.S.A., July 2006.

[20] T. Yoo and A. Goldsmith, "Sum-rate optimal multi-antenna downlink beamforming strategy based on clique search," in Proc. IEEE Global Tel. Conf. (GLOBECOM'05), St. Louis, MO, Dec. 2005, pp. 1510-1514.

[21] T.Yoo, N. Jindal, and A. Goldsmith, "Multi-Antenna Broadcast Channels with Limited Feedback and User Selection," to appear in IEEE Jour. Sel. Areas in Commun. (JSAC), 2007.

[22] M. Sharif and B. Hassibi, "On the capacity of MIMO broadcast channel with partial side information," IEEE Trans. Inform. Theory, vol. 51, no. 2, pp. 506-522, Feb. 2005.

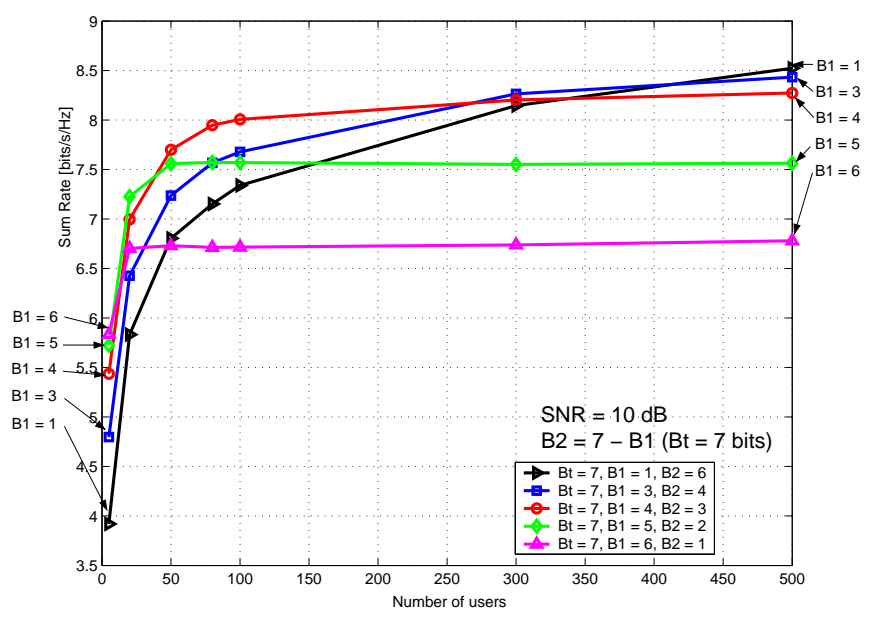

Fig. 2. Sum rate vs. number of users for $M=2$ and $\mathrm{SNR}=10 \mathrm{~dB}$.

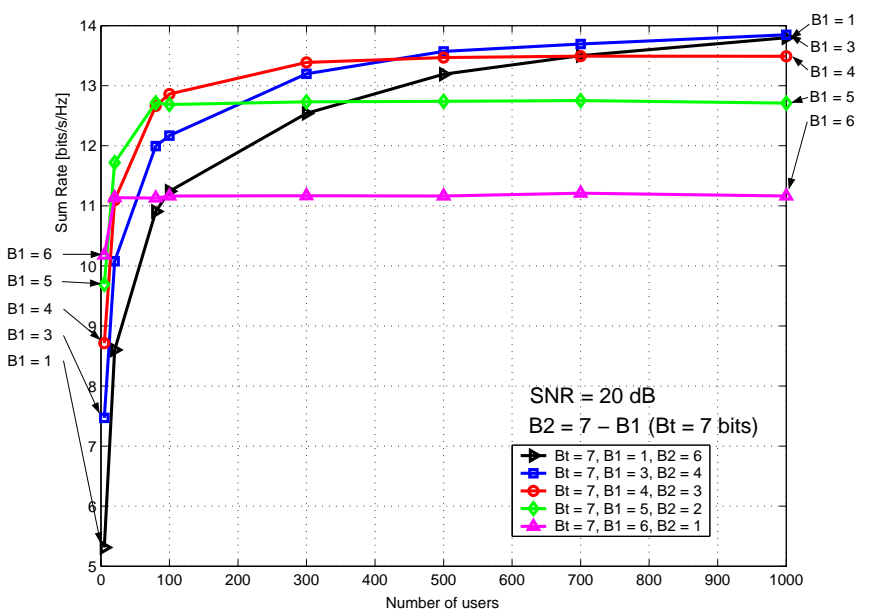

Fig. 3. Sum rate vs. number of users for $M=2$ and $\mathrm{SNR}=20 \mathrm{~dB}$.

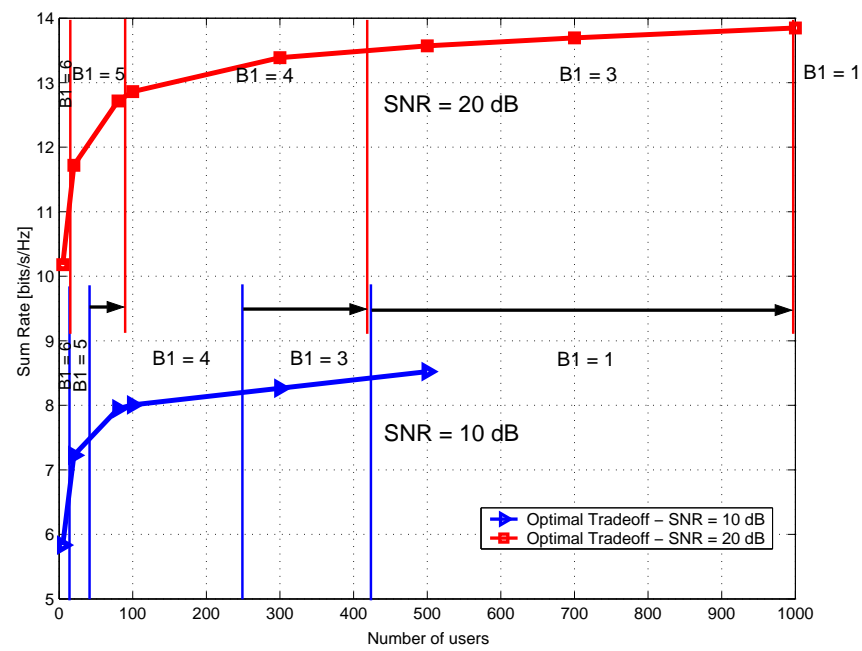

Fig. 4. Sum rate vs. number of users in a system with optimal $B_{1} / B_{2}$ balancing for different SNR values. 